\title{
Predicting intracranial hemorrhage after traumatic brain injury in low and middle-income countries: A prognostic model based on a large, multi-center, international cohort
}

\author{
Saleena Subaiya ${ }^{1 *}$, lan Roberts ${ }^{2}$ Edward Komolafe ${ }^{3}$ and Pablo Perel ${ }^{2}$
}

\begin{abstract}
Background: Traumatic brain injury (TBI) affects approximately 10 million people annually, of which intracranial hemorrhage is a devastating sequelae, occurring in one-third to half of cases. Patients in low and middle-income countries (LMIC) are twice as likely to die following TBI as compared to those in high-income countries. Diagnostic capabilities and treatment options for intracranial hemorrhage are limited in LMIC as there are fewer computed tomography (CT) scanners and neurosurgeons per patient as in high-income countries.

Methods: The Medical Research Council CRASH-1 trial was utilized to build this model. The study cohort included all patients from LMIC who received a CT scan of the brain $(n=5669)$. Prognostic variables investigated included age, sex, time from injury to randomization, pupil reactivity, cause of injury, seizure and the presence of major extracranial injury.

Results: There were five predictors that were included in the final model; age, Glasgow Coma Scale, pupil reactivity, the presence of a major extracranial injury and time from injury to presentation. The model demonstrated good discrimination and excellent calibration (c-statistic 0.71). A simplified risk score was created for clinical settings to estimate the percentage risk of intracranial hemorrhage among TBI patients.

Conclusion: Simple prognostic models can be used in LMIC to estimate the risk of intracranial hemorrhage among TBI patients. Combined with clinical judgment this may facilitate risk stratification, rapid transfer to higher levels of care and treatment in resource-poor settings.
\end{abstract}

Keywords: Neurosurgery, Trauma, Developing countries, Intracranial hemorrhage, Traumatic brain injury

\section{Background}

Traumatic brain injury (TBI) is a leading cause of death and disability worldwide, affecting approximately 10 million people annually according to the World Health Organization. This burden disproportionately affects low and middle-income countries (LMIC), with annual TBIrelated incidence rates of $150-170$ per 100,000 people as compared to the global rate of 106 per 100,000 [1]. Those in LMIC are twice as likely to die following severe TBI as compared to those in high-income countries [2].

\footnotetext{
* Correspondence: sas9141@nyp.org

'Department of Emergency Medicine, New York Presbyterian, New York, NY, USA

Full list of author information is available at the end of the article
}

Intracranial hemorrhage is a frequent and devastating sequelae of TBI, occurring between one-third to a half of cases [3,4]. Intracranial hemorrhage is the leading cause of death in lethally injured trauma patients accounting for $40-50 \%$ of fatalities [5] and results in a significant amount of long-term disability [6].

It has been suggested that organized emergency response systems and prompt transfer to trauma centers improve TBI patient morbidity and mortality [7]. An important adjunct to this is the availability of computed tomography (CT) scanners and neurosurgeons, with rapid surgical intervention resulting in a reduction in deaths [8]. CT scanning is the imaging modality of choice in the identification of intracranial hemorrhage due to its speed and diagnostic

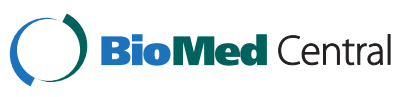


capabilities, however, there is only one scanner per 3.5 million people in low-income countries versus one per 64,900 in high-income countries [9]. There are also fewer neurosurgeons per patient, with one neurosurgeon per three million patients in Sub-Saharan Africa as compared to one per 20,000 in Europe [10]. Scarce resources in LMIC compounded with the increased burden of TBI make this a pressing public health issue.

Prognostic modeling provides a unique opportunity to aid clinical judgment and diagnostic ability, as they combine readily available patient data to predict the possibility of an outcome of interest [11,12]. The utility of these models in regards to TBI have shown to influence patient, next-of-kin and physician decision-making $[13,14]$. Additionally, they have been demonstrated to be more accurate than a physician's own predictive capabilities [13]. This can have a particularly important role in LMIC as there is a lack of specialty training in trauma among the healthcare workforce and diagnostic capabilities are limited $[12,15]$. The understanding and application of prognosis can be utilized in this setting to risk-stratify patients, and assist both care providers and family members with decisions to transfer patients to higher levels of care.

However, there is a paucity of prognostic models on TBI in LMIC, and no models currently exist that predict the risk of intracranial hemorrhage in this setting. The models that do exist suffer from multiple methodological flaws, including small sample sizes from a single center, inappropriate validation methods, and a lack of calibration or discrimination [16]. This highlights the necessity of new research to create accurate TBI prognostic modeling to aid clinicians with outcome prediction, as single factors do not have sufficient predictive value [17].

The Medical Research Council CRASH-1 (corticosteroid randomization after significant head injury) trial is the largest randomized controlled trial to date conducted in patients with TBI from $2005[18,19]$. The trial prospectively included patients within eight hours of injury, standardised their definitions of risk factors, and obtained CT scans of the head in over $75 \%$ of their patients. This allows for a large sample size to ensure high precision and valid prediction. Additionally, high recruitment of patients from LMIC allows for the identification of prognostic factors that are relevant to these settings. The results of this study demonstrated an association with corticosteroids and increased mortality of TBI patients. Prognostic models have been developed from this data to evaluate morbidity and mortality among TBI patients and have been externally validated in several settings; however, prediction of intracranial hemorrhage was not done $[3,20,21]$.

The purpose of our study is to identify readily available risk factors for intracranial hemorrhage, and build a clinically useful prognostic model for intracranial hemorrhage among TBI patients in LMIC that can be used by those without specialty training in neurosurgery or trauma.

\section{Methods}

\section{Selection of participants}

The study cohort was composed of all patients enrolled in the CRASH-1 trial from LMIC who received a CT scan of the brain. Adults aged 16 or older with TBI defined as any head injury with impaired consciousness (Glasgow coma score of 14 or less), and who were within eight hours of injury were eligible for inclusion in this trial [22]. The level of income of the country was defined as low, middle, or high- income countries as defined by the World Bank where middle was comprised with both low-middle and high-middle income [23]. Patients from low and middle-income countries were included in our analysis (Table 1).

\section{Outcomes}

CT scan diagnosis of intracranial hemorrhage was defined as the presence of subarachnoid hemorrhage, petechial hemorrhages, obliteration of third ventricle or basal cisterns, mid-line shift, evacuated hematomas and non-evacuated hematomas. These were dichotomized to include all those diagnosed by CT-scan to have intracranial hemorrhage, and those with a CT-scan who did not. Patients were administered a CT-scan based on the clinical judgment of their physician.

\section{Prognostic variables}

We considered age, sex, Glasgow Coma Scale (GCS), time from injury to randomization, pupil reactivity, cause of injury, seizure and whether the patient had sustained a major extracranial injury. These variables were all pre- and post- injury factors included in the CRASH-1 trial excluding hematemesis or melena, the presence of a wound infection, or pneumonia. These were selected for inclusion in our study as prior research has demonstrated a relationship between these variables and the presence of intracranial hemorrhage [24,25]. The analysis was adjusted for randomization to corticosteroids as this was related to increased mortality within the trial. We also assessed for the presence of interaction between treatment and potential prognostic factors as well as between prognostic factors for our model.

\section{Analyses}

All statistical analyses were conducted using STATA 10 (College Station, TX, USA). Univariate analysis was conducting using logistic regression modeling using the maximum likelihood theory to evaluate the relationship between prognostic variables and outcomes. We quantified each variable's predictive contribution by its $\mathrm{z}$ score (the model coefficient divided by its standard error). We 
Table 1 Low and middle-income countries as defined by the World Bank included in the CRASH trial

\begin{tabular}{|c|c|}
\hline Countries & Number of patients enrolled \\
\hline Albania & 41 \\
\hline Argentina & 359 \\
\hline Brazil & 119 \\
\hline Chile & 3 \\
\hline China & 87 \\
\hline Colombia & 832 \\
\hline Costa Rica & 20 \\
\hline Ecuador & 258 \\
\hline Egypt & 775 \\
\hline Georgia & 56 \\
\hline Ghana & 7 \\
\hline India & 973 \\
\hline Indonesia & 238 \\
\hline Iran & 233 \\
\hline Ivory Coast & 3 \\
\hline Kenya & 2 \\
\hline Malaysia & 176 \\
\hline Mexico & 17 \\
\hline Nigeria & 180 \\
\hline Pakistan & 17 \\
\hline Panama & 7 \\
\hline Paraguay & 10 \\
\hline Peru & 8 \\
\hline Romania & 319 \\
\hline Serbia & 23 \\
\hline South Africa & 366 \\
\hline Sri Lanka & 132 \\
\hline Thailand & 579 \\
\hline Tunisia & 63 \\
\hline Turkey & 2 \\
\hline Uganda & 43 \\
\hline Vietnam & 2 \\
\hline
\end{tabular}

graphically explored the relationship between age and intracranial hemorrhage, and GCS and intracranial hemorrhage to assess for linearity.

\section{Prognostic models}

The final model in multivariate analyses was built using backwards elimination, where all variables were initially included [26]. Variables were selected for elimination using a p-value of 0.05 , whereby a series of likelihood ratio tests with a p-value of $<0.10$ were utilized to determine which variables were kept in the final model. We explored for interaction between treatment and all other variables included in the final model using the likelihood ratio test. Ninety-five percent confidence intervals (CI) and p-values were calculated for all statistical tests of association. As there were few missing data, a complete case analysis was performed.

\section{Performance of the model}

The performance of the model was assessed through calibration and discrimination. Calibration was evaluated graphically by plotting the observed proportion of events against predicted risks for 10 risk groups of equal size, as well as statistically with the Hosmer-Lemeshow test. Discrimination of the model was assessed using the c-index.

\section{Internal validation}

The internal validity of the final model was assessed by the bootstrap re-sampling technique. Regression models were estimated in 50 models. For each of 50 bootstrap samples we refitted and tested the models on the original sample to obtain an estimate of predictive accuracy corrected for bias.

\section{Risk score estimation}

A clinical score was created using regression coefficients and a percentage risk calculated from these coefficients with an absolute risk equation. The absolute risk is expressed as a range of percentages for a given clinical score to facilitate its use in an emergency setting where the ability to do complex calculations may be limited.

\section{Ethics approval}

As this was a secondary retrospective analysis of the CRASH-1 trial and there were no patient identifiers utilized, there was no additional IRB approval that was obtained for conduction of this study. All MRC CRASH collaborators obtained local ethics and/or research committee approval for the original CRASH-1 trial.

\section{Results}

\section{General characteristics of study subjects}

Descriptive characteristics of study subjects are displayed in Table 2. A total of 5,669 TBI patients underwent a CT scan in low- and middle-income countries, and 3917 (69.1\%) were diagnosed with an intracranial hemorrhage. Among patients with intracranial hemorrhage, subarachnoid hemorrhage was present in 1900 (48.5\%), petechial hemorrhage in 1629 (41.6\%), hematomas not requiring evacuation in 1550 (39.6\%) and hematomas requiring evacuation in 808 (20.6\%).

There was an increased frequency of intracranial hemorrhage with increasing age. Males were more likely than females to have an intracranial hemorrhage. The risk of intracranial hemorrhage increased with increasing 
Table 2 Descriptive characteristics of study population

\begin{tabular}{|c|c|c|c|}
\hline Variables & $\mathrm{n}$ & n ICH & $\% \mathrm{ICH}$ \\
\hline Age (years) & 5669 & 3917 & 69 \\
\hline$>=19$ & 716 & 493 & 69 \\
\hline 20-29 & 1747 & 1147 & 66 \\
\hline 30-39 & 1187 & 819 & 69 \\
\hline $40-49$ & 863 & 597 & 69 \\
\hline $50-59$ & 580 & 412 & 71 \\
\hline 60-69 & 305 & 236 & 77 \\
\hline 70-79 & 201 & 157 & 78 \\
\hline \multirow[t]{2}{*}{$>=80$} & 70 & 56 & 80 \\
\hline & \multicolumn{3}{|c|}{$p^{1}<0.001$} \\
\hline Gender & 5669 & 3917 & 69 \\
\hline Male & 4723 & 3296 & 70 \\
\hline \multirow[t]{2}{*}{ Female } & 946 & 621 & 66 \\
\hline & \multicolumn{3}{|c|}{$p=0.012$} \\
\hline Time since injury (hour) & 5669 & 3917 & 69 \\
\hline$<=1$ & 1049 & 623 & 59 \\
\hline $1-\leq 3$ & 1677 & 1121 & 67 \\
\hline \multirow[t]{2}{*}{$>3$} & 2943 & 2173 & 74 \\
\hline & \multicolumn{3}{|c|}{$p<0.001$} \\
\hline GCS & 5669 & 3917 & 69 \\
\hline Mild (13-14) & 1294 & 641 & 50 \\
\hline Moderate (9-12) & 1825 & 1189 & 65 \\
\hline \multirow[t]{2}{*}{ Severe (3-8) } & 2550 & 2087 & 82 \\
\hline & \multicolumn{3}{|c|}{$p<0.001$} \\
\hline Pupil Reactivity & 5669 & 3917 & 69 \\
\hline Both reactive & 4741 & 3102 & 65 \\
\hline \multirow[t]{2}{*}{ One or both unreactive } & 928 & 815 & 88 \\
\hline & \multicolumn{3}{|c|}{$p<0.001$} \\
\hline Major Extracranial Injury & 5643 & 3897 & 69 \\
\hline None & 4281 & 2961 & 69 \\
\hline \multirow[t]{2}{*}{ Yes } & 1362 & 936 & 69 \\
\hline & \multicolumn{3}{|c|}{$p=0.758$} \\
\hline Type of injury & 5654 & 3909 & 69 \\
\hline Road traffic accident & 4144 & 2875 & 68 \\
\hline Fall $>2$ metres & 615 & 430 & 70 \\
\hline \multirow[t]{2}{*}{ Other mechanism } & 895 & 604 & 68 \\
\hline & \multicolumn{3}{|c|}{$p=0.998$} \\
\hline Seizure & 5646 & 3900 & 69 \\
\hline No & 5216 & 3603 & 69 \\
\hline \multirow[t]{2}{*}{ Yes } & 430 & 297 & 69 \\
\hline & \multicolumn{3}{|c|}{$p=0.998$} \\
\hline Death at Two Weeks & 5669 & 3917 & 69 \\
\hline Alive & 4386 & 2793 & 64 \\
\hline \multirow[t]{2}{*}{ Dead } & 1283 & 1124 & 88 \\
\hline & \multicolumn{3}{|c|}{$p<0.001$} \\
\hline
\end{tabular}

Table 2 Descriptive characteristics of study population (Continued)

\begin{tabular}{llll}
\hline Death at Six Months & 5443 & 3762 & 69 \\
Alive & 3890 & 2410 & 62 \\
Dead & 1553 & 1352 & 87 \\
& $p<0.001$ & & \\
\hline
\end{tabular}

${ }^{1} p$ values represent significance testing for univariate odds ratios.

time from injury to presentation. The presence of an intracranial hemorrhage was associated with death at both two weeks $(x 2=266.1, \mathrm{df}=1, \mathrm{p}<0.001)$, and at 6 months $(x 2=327.7, \mathrm{df}=1, \mathrm{p}<0.001)$.

The relationship between ten-year age categories and $\log$ odds of intracranial hemorrhage was linear, and therefore analysed as an ordered categorical variable. The relationship between GCS and log odds of intracranial hemorrhage was closely linear, and was therefore analysed as a continuous variable in multivariable analysis.

\section{Multivariable predictive models}

There were five predictors that were included in the final model: age, GCS, pupil reactivity, the presence of a major extracranial injury and time from injury to presentation (Table 3). GCS was the strongest predictor, followed by time from injury to presentation, and age. The presence of a major extra-cranial injury was associated with a reduction in the risk of having an intracranial hemorrhage (ICH).

\section{Performance of the model}

The model showed good discrimination, with a c-statistics of 0.71. It demonstrated good calibration graphically and after evaluation with the Hosmer-Lemeshow test (Figure 1).

\section{Internal validation}

We did not find evidence of a significant overoptimism in our model development. The overoptimism for the c-statistic with the bootstrapping procedure was $0.15 \%$.

\section{Clinical risk score}

Individual risk scores can be calculated from Table 4 and are associated with a corresponding risk percentage (Table 5). For example, the risk of intracranial hemorrhage in a 55-year-old TBI patient with a GCS score of 12 , reactive pupils, no major extracranial injury who presents 3 hours after injury would have a calculated risk score of 14 which corresponds with an $60-<65 \%$ risk of intracranial hemorrhage. 
Table 3 Multivariable predictive model

\begin{tabular}{lcc}
\hline Exposure variables & \multicolumn{2}{c}{ Low and middle income countries } \\
\cline { 2 - 3 } & $\begin{array}{l}\text { Adjusted odds ratio } \\
\left(\mathbf{9 5 \%} \mathbf{C l}^{\mathbf{1}}\right), \mathbf{z} \text {-score }\end{array}$ & p-value $\mathbf{2}^{\mathbf{2}}$ \\
\hline Age $^{3}$ & $1.10(1.06,1.15), 5.19$ & $<0.001$ \\
Glasgow Coma Scale $^{4}$ & $1.21(1.18,1.23), 17.46$ & $<0.001$ \\
One or both pupils unreactive & $1.81(1.44,2.26), 5.14$ & $<0.001$ \\
Major extracranial injury & $0.78(0.68,0.90),-3.35$ & 0.001 \\
Time since injury & $1.38(1.27,1.48), 8.19$ & $<0.001$ \\
\multicolumn{1}{c}{ c-index: 0.71} & &
\end{tabular}

${ }^{1}$ Ninety-five percent confidence interval.

${ }^{2}$ Wald Test $p$-value for association between variables and outcome.

${ }^{3}$ OR for a 10-year increase in age from a baseline group of $<16$.

${ }^{4}$ OR for a one-unit decrease in GCS from a baseline of 14 .

${ }^{5} \mathrm{OR}$ for a categorical increase in hours of injury until randomization (baseline $<1 \mathrm{hr}, 1-\leq 3 \mathrm{hrs},>3 \mathrm{hrs}$ ).

\section{Discussion}

We have developed a prognostic model utilizing readily available clinical data to predict the risk of intracranial hemorrhage in TBI patients from LMIC. The model has demonstrated good discrimination, excellent calibration and has been internally validated.

Advanced age, GCS, pupil reactivity, the presence of a major extracranial injury and time from injury to presentation were all found to be predictors for intracranial hemorrhage (ICH). GCS demonstrated a linear relationship with increased risk for intracranial hemorrhage, except for those with a calculated score of three. This could be attributed to those patients that have been sedated and intubated prior to recording of GCS, as these are given a score of three by default [27]. A linear relationship between advanced age and increased risk of poor outcome after TBI has been documented previously and was demonstrated in our study [24]. The increasing risk of hemorrhage with increasing time from injury to presentation may reflect the fact that slower bleeds are more likely to be detected at a later scan and could have been missed in early imaging. This can also

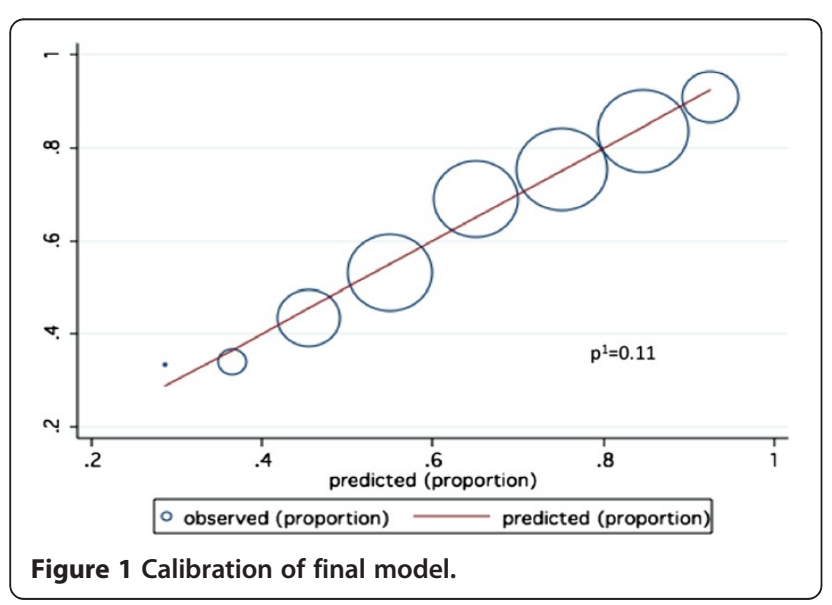

Table 4 Estimation of the risk score of intracranial hemorrhage

\begin{tabular}{|c|c|}
\hline Variable & Risk score $^{1}$ \\
\hline \multicolumn{2}{|c|}{ Age (years) } \\
\hline$>=19$ & 0 \\
\hline $20-29$ & 1 \\
\hline $30-39$ & 2 \\
\hline $40-49$ & 3 \\
\hline $50-59$ & 4 \\
\hline $60-69$ & 5 \\
\hline $70-79$ & 6 \\
\hline$>=80$ & 7 \\
\hline \multicolumn{2}{|l|}{$\overline{G C S}$} \\
\hline 14 & 0 \\
\hline 13 & 2 \\
\hline 12 & 4 \\
\hline 11 & 6 \\
\hline 10 & 8 \\
\hline 9 & 10 \\
\hline 8 & 12 \\
\hline 7 & 14 \\
\hline 6 & 16 \\
\hline 5 & 18 \\
\hline 4 & 20 \\
\hline 3 & 22 \\
\hline
\end{tabular}

\section{Pupil Reactivity}

Both reactive 0

One or both unreactive 0

Major Extracranial Injury

None $\quad 0$

Yes -3

Time since injury (hour)

$<=1 \quad 0$

$1-\leq 3 \quad 3$

$>3 \quad 6$

${ }^{1}$ Risk Score estimated from regression coefficients stratified by prognostic variable. Values have been rounded up to nearest whole number to facilitate operator use.

be attributed to prolong extrication times, which has been demonstrated to be associated with major injury [25]. Additionally the possibility of bias must be considered, as patients referred for more serious injury may be more likely to present with a bleed. Also a change in neurological status or development of new clinical symptoms may prompt patients to seek delayed care after injury.

This study has limitations. In order to explore the generalisability of a prognostic model to a similar patient 
Table 5 Percentage risk of intracranial hemorrhage according to the risk score

\begin{tabular}{lc}
\hline Risk score & Percentage risk $^{\mathbf{1 , 2}}$ \\
\hline$-3-<0$ & $25-<30$ \\
$0-<3$ & $30-<35$ \\
$3-<5$ & $35-<40$ \\
$5-<7$ & $40-<45$ \\
$7-<9$ & $45-<50$ \\
$9-<11$ & $50-<60$ \\
$11-<15$ & $60-<65$ \\
$15-<17$ & $65-<70$ \\
$17-<20$ & $70-<75$ \\
$20-<23$ & $75-<80$ \\
$23-<26$ & $80-<85$ \\
$26-<31$ & $85-<90$ \\
$31-<39$ & $90-<95$
\end{tabular}

${ }^{1}$ Percentage risk calculated from risk score utilizing the following equation: Percentage risk $=100 *\left(\mathrm{e}^{(0.1 * \text { score })} /\left(1+\mathrm{e}^{\left(0.11^{*} \text { score }\right)}\right)\right)$.

${ }^{2}$ Percentage risk $<50 \%$ has an margin of error of $1-1.5 \%$ points secondary to rounding of risk score.

population within a different setting, external validation is necessary [28]. However, we did not have access to data that contains the patient population and variables included in this study, so external validation was not possible. Another limitation is the consideration of all intracranial hemorrhage together as variability in prognostic factors may exist depending on type of bleed. The use of CT scanning to diagnose intracranial hemorrhage across different centers is subject to interobserver variability however, because this potential measurement error is unrelated to prognostic factors the estimate of the prognostic factor remains unbiased, although potentially imprecise [29]. The exclusion of patients who did not have a CT scan is an additional limitation that may cause potential selection bias in our sample.

The strength's of this study is the use of prospective, standardised data collection on prognostic variables, and a well-defined patient cohort with few losses to follow-up. Additionally, this is the largest sample of patients from LMIC with TBI to derive a prognostic model to our knowledge.

\section{Conclusion}

This is the first study of its kind, to our knowledge, to provide a risk stratification of intracranial hemorrhage among TBI patients involving multiple prognostic variables. While other studies have evaluated prognostic variables to triage and treat trauma patients such as the New Orleans Criteria, The Canadian Head CT Rule, and the Trauma Score and Injury Severity Score, no risk score has been designed to specifically evaluate the risk of intracranial hemorrhage in TBI patients from LMIC
[30-34]. The scores that do exist in this setting focus on morbidity and mortality after head injury and all suffer from methodological limitations [17].

Prognostic factors in TBI are often used within the context of clinical judgment and radiographic evidence to diagnose intracranial hemorrhage in patients. However, the utility of a single prognostic variable is limited and a combination of variables into a prognostic model could be a more useful clinical tool. While a prognostic model should never replace clinical judgment, it can be used in conjunction with professional knowledge to inform decision-making. Previous studies have demonstrated that prognostic modeling in TBI can be used to accurately access long-term outcomes [20]. Within LMIC this can be useful for diagnosis, referral, and treatment. However, although a prognostic model could help the decision making process and ensure a more rational use of limited resources, increase in TBI related resources (CT scan and neurosurgeons) in this setting is paramount to prevent long-term disability and mortality.

In summary, this model within this population demonstrated good performance; however, future research utilizing a prospective cohort design to perform external validation is needed. Further investigations should assess if the application of this risk score in a low-income settings would improve patients' outcomes. While it would be worthwhile to determine a risk score for patients who had a neurosurgical intervention, inherent bias may flaw these studies, as physicians may be influenced to operate based on variables included in the model.

\section{Abbreviations}

TBI: Traumatic brain injury; ICH: Intracranial hemorrhage; LMIC: Low and middleincome countries; CT: Computed tomography; GCS: Glasgow coma scale. There was no grant funding or other financial support involved in this study. The original founders of the CRASH trial had no role in this study design, data collection and analysis, decision to publish, or preparation of the manuscript. The original funding for the $\mathrm{CRASH}-1$ trial was obtained from the UK Medical Research Council.

\section{Competing interests}

There are no financial, personal or professional interests that could be construed to have Influenced this paper.

\section{Authors' contributions}

SS, PP, and IR conceived the study. SS and PP created the statistical analysis plan and analyzed the data. EK provided key insight in creating an accessible and user-friendly risk score. SS drafted the manuscript, and all authors contributed substantially to its revision.

\section{Author details}

${ }^{1}$ Department of Emergency Medicine, New York Presbyterian, New York, NY, USA. ${ }^{2}$ CRASH Trials Coordinating Center, The London School of Hygiene and Tropical Medicine, London, UK. ${ }^{3}$ Department of Neurosurgery, Obafemi Awolowo University, Ife Ife, Nigeria.

Received: 5 August 2012 Accepted: 12 November 2012 Published: 19 November 2012

\section{References}

1. Hyder AA, et al: The impact of traumatic brain injuries: a global perspective. NeuroRehabilitation 2007, 22(5):341-353. 
2. De Silva MJ, et al: Patient outcome after traumatic brain injury in high-, middle- and low-income countries: analysis of data on 8927 patients in 46 countries. Int J Epidemio/ 2009, 38(2):452-458.

3. Perel $P$, et al: Intracranial bleeding in patients with traumatic brain injury: a prognostic study. BMC Emerg Med 2009, 9:15.

4. Servadei F, et al: Traumatic subarachnoid hemorrhage: demographic and clinical study of 750 patients from the European brain injury consortium survey of head injuries. Neurosurgery 2002, 50(2):261-267. discussion 267-269.

5. Kauvar DS, Wade CE: The epidemiology and modern management of traumatic hemorrhage: US and international perspectives. Crit Care 2005, 9(Suppl 5):S1-S9.

6. Corrigan JD, Selassie AW, Orman JA: The epidemiology of traumatic brain injury. J Head Trauma Rehabil 2010, 25(2):72-80.

7. Ghajar J: Traumatic brain injury. Lancet 2000, 356(9233):923-929.

8. Seelig JM, et al: Traumatic acute subdural hematoma: major mortality reduction in comatose patients treated within four hours. $N$ Engl J Med 1981, 304(25):1511-1518

9. WHO: Global forum to improve developing country access to medical devices, in World Health Organization Media Centre. Geneva: World Health Organization Geneva; 2010.

10. Aarli JA, Diop AG, Lochmuller H: Neurology in sub-Saharan Africa: a challenge for world federation of neurology. Neurology 2007, 69(17):1715-1718.

11. Wagner $A K$, et al: Intentional traumatic brain injury: epidemiology, risk factors, and associations with injury severity and mortality. J Trauma 2000, 49(3):404-410

12. Le TH, Gean AD: Neuroimaging of traumatic brain injury. Mt Sinai J Med 2009, 76(2):145-162

13. Honeybul S, O'Hanlon S, Ho KM: Decompressive craniectomy for severe head injury: does an outcome prediction model influence clinical decision-making? J Neurotrauma 2011, 28(1):13-19.

14. Bandiera G, et al: The Canadian C-spine rule performs better than unstructured physician judgement. Ann Emerg Med 2003, 42(3):395-402.

15. Razzak JA, Kellermann AL: Emergency medical care in developing countries: is it worthwhile? Bull World Health Organ 2002, 80(11):900-905.

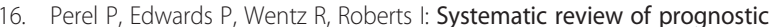
models in traumatic brain injury. BMC Med Infor Decis Making 2006 38:1-10

17. Mushkudiani NA, et al: A systematic review finds methodological improvements necessary for prognostic models in determining traumatic brain injury outcomes. J Clin Epidemiol 2008, 61(4):331-343.

18. Roberts I, et al: Effect of intravenous corticosteroids on death within 14 days in 10008 adults with clinically significant head injury (MRC CRASH trial): randomised placebo-controlled trial. Lancet 2004, 364(9442):1321-1328.

19. Crash Trial Collaborators: Final results of MRC CRASH, a randomised placebo-controlled trial of intravenous corticosteroid in adults with head injury-outcomes at 6 months. Lancet 2005, 365(9475):1957-1959.

20. Honeybul S, et al: The retrospective application of a prediction model to patients who have had a decompressive craniectomy for trauma. J Neurotrauma 2009, 26(12):2179-2183.

21. Perel $P$, et al: Predicting outcome after traumatic brain injury: practical prognostic models based on large cohort of international patients. BMJ 2008, 336(7641):425-429.

22. The Crash trial management group: The CRASH trial protoco (Corticosteroid randomisation after significant head injury) [ISRCTN74459797]. BMC Emerg Med 2001, 1(1):1.

23. The World Bank: World development indicators. Washington, DC: World Bank; 2006.

24. Hukkelhoven CW, et al: Patient age and outcome following severe traumatic brain injury: an analysis of 5600 patients. J Neurosurg 2003, 99(4):666-673.

25. Palanca Sylvia EA: Mechanisms of motor vehicle accidents that predict major injury. Emerg Med Australas 2003, 15(5-6):423-428.

26. Royston $P$, et al: Prognosis and prognostic research: Developing a prognostic model. BMJ 2009, 338:b604.

27. Marion DW, Carlier PM: Problems with initial Glasgow Coma Scale assessment caused by prehospital treatment of patients with head injuries: results of a national survey. J Trauma 1994, 36(1):89-95.

28. Altman DG, et al: Prognosis and prognostic research: validating a prognostic model. BMJ 2009, 338:b605.
29. Laalo JP, et al: Reliability of diagnosis of traumatic brain injury by computed tomography in the acute phase. J Neurotrauma 2009, 26(12):2169-2178.

30. Haydel MJ, et al: Indications for computed tomography in patients with minor head injury. N Engl J Med 2000, 343(2):100-105.

31. Stiell IG, et al: The Canadian CT Head Rule for patients with minor head injury. Lancet 2001, 357(9266):1391-1396.

32. Pal J, Brown R, Fleiszer D: The value of the Glasgow Coma Scale and Injury Severity Score: predicting outcome in multiple trauma patients with head injury. J Trauma 1989, 29(6):746-748.

33. Champion HR, et al: A revision of the trauma score. J Trauma 1989, 29(5):623-629

34. Kumar B, Paul G, Sharma AK: Injury Severity Score (ISS) as a yardstick in assessing the severity and mortality of various abdomino-pelvic trauma hospitalized victims-a clinical vis-a-vis autopsy study. Med Sci Law 1989, 29(4):333-336

doi:10.1186/1471-227X-12-17

Cite this article as: Subaiya et al:: Predicting intracranial hemorrhage after traumatic brain injury in low and middle-income countries: $\mathrm{A}$ prognostic model based on a large, multi-center, international cohort. BMC Emergency Medicine 2012 12:17.

\section{Submit your next manuscript to BioMed Central and take full advantage of:}

- Convenient online submission

- Thorough peer review

- No space constraints or color figure charges

- Immediate publication on acceptance

- Inclusion in PubMed, CAS, Scopus and Google Scholar

- Research which is freely available for redistribution
C Biomed Central 